\title{
Spiritual care rendered by registered nurses at a state hospital in Windhoek Namibia
}

\begin{abstract}
The aim of this study was to determine the spiritual care rendered by nurses to hospitalized patients and its effectiveness at a state hospital in Windhoek, Namibia. The objectives of this study were to assess the understanding of registered nurses on spiritual care, describe spiritual care given to patients by registered nurses and to determine the effectiveness of spiritual care given to patients by registered nurses. A quantitative descriptive research design was used for this study to survey the registered nurses. Data were collected using the closed ended questions questionnaires. The target population was 40 registered nurses on duty. 40 questionnaires were distributed to participants and $32(80 \%)$ questionnaires were returned and analyzed. Data are presented in tables and figures. The findings of this study show that registered nurses do understand the importance of spiritual care to patient's wellbeing. Nurses understand that the spiritual care is part of holistic care. They understand that it is their role to render spiritual care as part of holistic care. However, the findings of this study shows that nurses rarely render spiritual care to patients.
\end{abstract}

Volume 5 Issue 4 - 2018

Rainhold Vatilifa Ndaikile, Hans Justus

Amukugo, Anna Panduleni Kauko Shilunga, Abel Karera

Faulty of Health Sciences, School of Nursing, University of Namibia, Namibia

Correspondence: Hans Justus Amukugo, Faulty of Health Sciences, School of Nursing, University of Namibia, Namibia, Email hamukugo@unam.na

Received: July 12, 2018 | Published: August 02, 2018

Keywords: spiritual care, nurses, spirituality, holistic care

\section{Introduction}

Holistic care is care of the mind, body and soul or spirit. ${ }^{1}$ There is a body of evidence in nursing and health care literature to suggest that spirituality is integral to patients' well-being. ${ }^{2}$ The holistic approach to care is based on the premise that caring for the whole person constitutes the body, mind and spirit. Nightingale with her modern nursing has also emphasized on spiritual care by saying that the needs of the spirit are as critical to health as those individual organs which make up the body. We have all observed that a physical condition can affect the mind and spirit. ${ }^{3}$ We are also aware that when a person is hurting emotionally or spiritually, all sorts of physical ailments may manifest. ${ }^{3}$ The biblical world understanding has always maintained a unified view of the person where God's action relates to the whole person.

Spiritual care is an essential component in the nursing context, as nurses have to care for patients who may often turn to the spiritual dimension to cope and heal. ${ }^{2}$ These aspects are important issues to be considered in planning what should be taught as part of spiritual care. They reiterated that it has been argued that a failure to incorporate spirituality into nursing care by not addressing the spiritual needs of patients is unethical as spirituality is a part of being human. Lack of spiritual care service to the patients deprives the patient's right to holistic care. Several authors have noted that nursing education has provided few opportunities for the inclusion of spirituality and spiritual care. ${ }^{4,5}$ This void leaves the nurse unprepared to meet the challenges of providing therapeutic spiritual care for patients and their families. There is a need for spirituality and spiritual care activities to be included in the nursing curricula. ${ }^{5}$ A study to determine the type of spiritual care being given to patients by registered nurses becomes important in understanding the holistic care being accorded to patients.

The focus on spiritual care continues to grow especially for inpatient's care. Research on health, well-being and spirituality has grown in the Western context, with studies showing that spirituality is an important part of a patient's life when confronted with illness.
There is a body of evidence in nursing and health care literature to suggest that spirituality is integral to patient's well-being. ${ }^{3}$ The holistic approach to care is based on the premise that caring for the whole person constitutes the body, mind and spirit. Many nurses have difficulty to understand and integrate spiritual care into practice and consequently neglect this aspect of care. ${ }^{7}$ In order to meet the needs of patients holistically and to assist them in making sense of their circumstances, spiritual nursing care should be considered as equally important as physical, emotional and social care dimensions. ${ }^{7}$ In spite of this interest in spirituality, there researcher has found no studies that have been recorded in Namibia on spirituality as it relates to patient care.

The aim of this study was to determine and describe the spiritual care being rendered by nurses to hospitalized patients and its effectiveness at a State Hospital in Windhoek Namibia.

\section{Methodology}

A quantitative, descriptive research design was used to describe the understanding of professional nurses on spiritual care, the type of spiritual nursing care accorded by professional nurses to patients at a state hospital in Windhoek and the effectiveness of that spiritual care. The population was registered nurses working in in-patients wards at a state hospital in Windhoek. The total population size was 40 registered nurses. All members of the population were selected to take part in the study. Data was collected using self-administered survey questionnaires mainly comprising of closed-ended Likert type of questions offering the professional nurse response options from which they could choose the one that most closely matched the appropriate answer. The questionnaire was developed according to the aims and objectives of the study and comprised of four sections. Part A focused on the demographic and various personal and professional background variables. Part B focused on the understanding of spiritual nursing care by participants using five point Likert scale. Part C solicited the type of nursing care given to patients and part $\mathrm{D}$ focused 
on the effectiveness of spiritual care rendered by registered nurses. Data were coded and analyzed using Microsoft excel. Frequency distributions were calculated for each individual variable and mean score were generated for each objective. Results were displayed in frequencies and percentages.

Permission to conduct the research was sought and granted from University of Namibia and the Ministry of Health and Social Services, Namibia. Furthermore, access to participants was granted by the nursing managers and individual nurses voluntarily participated by means of signing an informed consent form. All rights of the participants were respect in accordance with principles of research ethics.

\section{Results}

\section{Demographic characteristics of the sample}

A total of 40 questionnaires were distributed and 32 were returned giving $80 \%$ response rate. Age and gender distribution of the sample is show in Table 1 . The majority of the participants were aged between 20 and 30 years $(56.25 \%)$ and predominantly female $(81.25 \%)$. Nursing work experience of the participants ranged from 0-10 years $(50 \%), 10-20$ years $(25 \%), 21-30$ years, $(6.25 \%), 31-40$ years $(12.5 \%)$ and above 41 years $(6.25 \%)$.

Table I Gender and age distribution

\begin{tabular}{llll}
\hline Age (year) & Gender & & Total \\
& Male & Female & \\
\hline $20-30$ & $\mathrm{n}=6(18.75 \%)$ & $\mathrm{n}=12(37.5 \%)$ & $\mathrm{n}=18(56.25 \%)$ \\
$3 I-40$ & $\mathrm{n}=0(0 \%)$ & $\mathrm{n}=6(18.75 \%)$ & $\mathrm{n}=6(18.75 \%)$ \\
$4 I-50$ & $\mathrm{n}=0(0 \%)$ & $\mathrm{n}=2(6.25 \%)$ & $\mathrm{n}=2(6.25 \%)$ \\
$5 I-60$ & $\mathrm{n}=0(0 \%)$ & $\mathrm{n}=6(18.75 \%)$ & $\mathrm{n}=6(18.75 \%)$ \\
Over 60 & $\mathrm{n}=0(0 \%)$ & $\mathrm{n}=0(0 \%)$ & $\mathrm{n}=0(0 \%)$ \\
$\%$ of total & $18.75 \%$ & $8 I .25 \%$ & $100 \%$ \\
\hline
\end{tabular}

\section{Spiritual participation}

Participants were asked their frequency of participation in spiritual activities during training. All the participants indicated that they participated in spiritual activities with the majority (40\%) participating once a week. Thirty three percent and $27 \%$ of the participants attended spiritual activities several times a week and once a month respectively. No participants were attending to spiritually activities daily or once a year. Christianity was the most common faith practiced by participants (88\%) followed by Muslin and Atheist (6\% respectively). No other religion/faith was being practiced by the participants.

\section{Understanding of registered nurses on spiritual care}

The participants were asked to rate their understanding concerning spiritual care using a five point Likert scale. High number of participant responded positively toward the understanding of the Spirituality. Overall, participants agreed that spiritual care is part of holistic care $(87.5 \%)$, rendering spiritual care helps patients' recovery $(80 \%)$ and that professional nurses have the responsibility to ensure spiritual care is accorded to patients (50\%). Participants also disagreed that spiritual care is for the church $(81.25 \%)$, that spiritual care is not a necessary part of patient $(75 \%)$, that spiritual care is for pastors or religious leaders $(81.25 \%)$ and that spiritual care is not for nurses $(81.25 \%)$. More expanded findings are detailed in Figure 1.

\section{Spiritual care given to patients by registered nurses}

Participants were asked to rate how often they render the spiritual care activities during the caring of patients. The four choices were given to participants ranging from never, rarely, sometimes and often. Overall, $50 \%$ indicated that they sometimes or often gather information on the patient's spiritual background. Participants rarely $(28.57 \%)$ or never $(28.57 \%)$ assess patient's spiritual interests. The majority never pray or meditate with their patients $(46.67 \%)$ or help a patient reflect on his/her beliefs about death $(50 \%)$. Participants indicated that they never $(43.75 \%)$ or rarely $(6.25 \%)$ refer a patient for spiritual counseling. Detailed findings are highlighted in Table 2.

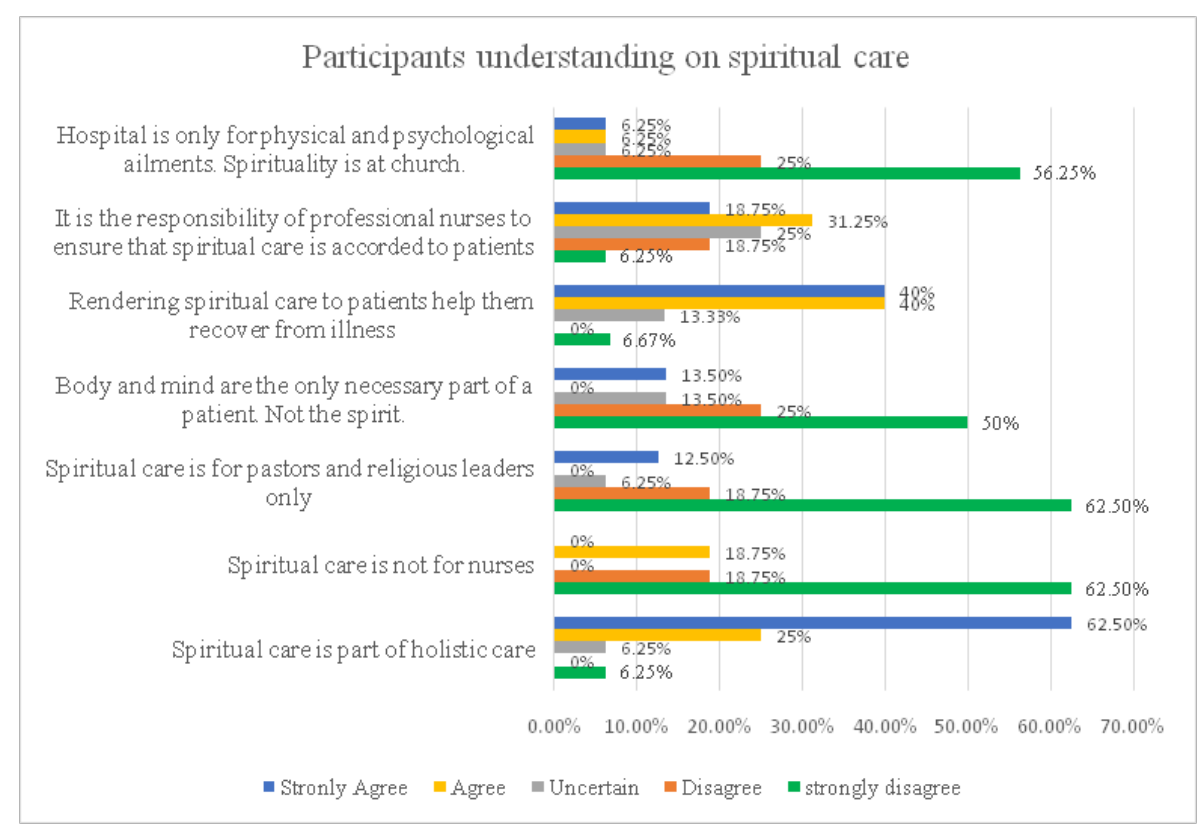

Figure I Understanding of spiritual care. 
Table 2 Spiritual care rendered to patients

\begin{tabular}{|c|c|c|c|c|}
\hline Aspect of spiritual care & Never & Rarely & Sometimes & Often \\
\hline Gather information on the patient's spiritual background. & $37.5 \%$ & $12.5 \%$ & $31.25 \%$ & $18.75 \%$ \\
\hline Assess a patient's spiritual interest. & $28.57 \%$ & $28.57 \%$ & $21.43 \%$ & $21.43 \%$ \\
\hline Recommend spiritual books or writings. & $37.5 \%$ & $6.25 \%$ & $43.75 \%$ & $12.5 \%$ \\
\hline Pray or meditate with a patient. & $46.67 \%$ & $13.33 \%$ & $40 \%$ & $0 \%$ \\
\hline Recommend meditation. & $26.67 \%$ & $20 \%$ & $40 \%$ & $13.33 \%$ \\
\hline $\begin{array}{l}\text { Refer a patient to others for spiritual counseling, e.g. minister, priest, rabbi, Chaplain or } \\
\text { traditional healer. }\end{array}$ & $43.75 \%$ & $6.25 \%$ & $31.25 \%$ & $18.75 \%$ \\
\hline Recommend spiritual forgiveness, confession. & $31.25 \%$ & $12.5 \%$ & $32.25 \%$ & $25 \%$ \\
\hline Assist a patient to talk about their personal spiritual beliefs or practice. & $18.75 \%$ & $31.25 \%$ & $43.75 \%$ & $6.25 \%$ \\
\hline Help a patient reflect on his/her beliefs about what happens after death? & $43.75 \%$ & $18.75 \%$ & $31.25 \%$ & $6.25 \%$ \\
\hline Help a patient reflect on his/her beliefs about death. & $50 \%$ & $18.75 \%$ & 18.75 & $12.5 \%$ \\
\hline
\end{tabular}

\section{The effectiveness of spiritual care}

Participants were asked rate the effect of spiritual care (when it was provided) on patient prognosis. The majority rated this as good $(62.5 \%)$, whilst others rated the effect as improving $(6.25 \%)$ or fair $(6.25 \%)$ and the same as other patients (18.75\%). There was no response given by $6.25 \%$ of the sample. The majority of the participants $(92.86 \%)$ reported that patients showed appreciation of the spiritual care rendered whilst $7.14 \%$ did not. Of those patients who received spiritual care, $80 \%$ were reported to have passed away with only $20 \%$ recovering from illness. Those who passed away were reported to have died a peaceful death $(61.54 \%)$ whilst $38.46 \%$ died in the same manner as those who did not receive spiritual care.

\section{Discussion}

The provisions of spiritual care have long been documented as necessary in rendering holistic patient care. An earlier study however reported that lack of guidance both during training and in practice on how to provide such care presents a barrier in practice. ${ }^{7}$ Even though content on spiritual care is available in most nursing curriculums, nurses have been reported to struggle in teaching such content and integrating it in clinical practice. ${ }^{8}$ Similar sentiments were also echoed in a previous study where lack of clear guidelines, limited training and professional development regarding spiritual care were reported as the reasons for the negletion of spiritual care in clinical practice. ${ }^{9}$

\section{Demographic findings}

Majority of the participants were young, between the ages of 2030 years. Most of the registered nurses enroll for training straight from school and enter into practice at a relatively young age. This is also reflected in the level of experience where the majority where below 10 years of experience. Understanding of spirituality and its application requires a certain level of maturity in an individual. The relatively you population of nurses at this hospital can be assumed to be more focused on integrating knowledge of the physical and mental wellbeing into clinical practice with little attention on the spiritual care necessary for provision of holistic care. The majority of the participants were females $(81.25 \%)$ with a female to male ratio of approximately 1:4. These findings are consisted with the findings of other studies where females are dominating in the nursing profession. ${ }^{2}$ Nursing was traditionally regarded as a predominantly female profession and interest by males is continually growing that may balance this historical gender biased in the future. ${ }^{2}$

In this study, participants showed a high level of participation in religious or spiritual activities with all participants committing to either several times a week, once a week or once a month. Religious affiliation is regarded as a necessary part of societal integration of individual upbringing in the local settings. Children are oriented into religion at a tender age and this trend is maintained into adolescents and young adult life as reflected in the young nursing professionals in this sample. The personal spiritual participation of nurses may play an important role in improving their attitudes toward spiritual care and their professional commitment and caring capabilities. This is similar to one of the main findings of a related study which reported positive relationship between nurses' spiritual health and attitudes towards spiritual care. ${ }^{10}$ Nurses who have better spiritual participation may be more sensitive to patients' spiritual difficulties and needs because they understand the meaning of spirituality and may be able to relate to the specific spiritual difficulties that their patients are dealing with.

\section{Understanding of registered nurses on spiritual care}

The majority $(87.5 \%)$ of the participants overall agreed that spiritual care is part of the holistic care. They understood that the patient is constituted of the body, mind and spirit. This can be attributed to their own religious and spiritual practice as all the participants were engaged in spiritual/religious activities. These findings are of one accord with an earlier study which reported that holistic care (body, mind and spirit) have long been recognized by the healing professionals and the three aspects must be considered in order to effectively maintain or restore health. ${ }^{11}$ A study among Irish nurses also echoed the same findings, where the majority, $77 \%$ and $75 \%$, reported that spiritual care was a part of their role and a key component of holistic nursing care respectively. ${ }^{12}$ In this study, the majority of the participant showed an understanding of the spiritual care and its relevance to nursing care. They also accept that it is their role to render spiritual care to patients. A majority $(81.25 \%)$ in this study denied that spiritual care is for pastors and religious leaders only 
$80 \%$ of the participants understand that spiritual care helps the patient recover from illnesses. They understand that spiritual care is important to patient's wellbeing. Similar findings were also reported previously among nurses in Jordan where the majority indicated high levels of perceptions of spirituality and spiritual care. ${ }^{13}$ They also reported that the majority of nurses in their study were spiritually oriented even without any education on spirituality and the reiterated the importance of spiritual care being given to their patients. Nurses by the nature of their training and practice, usually possess an acceptable level of understanding regarding spirituality and spiritual care.

\section{Spiritual care given to patients by registered nurses}

Unlike other previous studies on spiritual care,,$^{2,7,14}$ the majority of the participants in this study indicated that they rarely or never render spiritual care. Spiritual care such as gathering of information on the patient's spiritual background, assessment of a patient's spiritual interest, recommendation of spiritual books or writings, praying or meditating with a patient, recommending meditation, referring a patient to others for spiritual counseling, e.g., minister, priest, rabbi, chaplain or traditional healer and recommending spiritual forgiveness, confession and assisting a patient to talk about their personal spiritual beliefs or practice are some of the activities assessed in this study. These activities can form a baseline or introduction of nursing spiritual care in Namibia. Participants in a previous study reported that they don't always feel prepared to offer spiritual support for patients. ${ }^{3}$

There is a big gap between the understanding of spiritual care by the participants, their role in it, its importance and the spiritual care rendered to patients. Of the $80 \%$ of participants who indicated that spiritual care helps patient to recover and $50 \%$ that indicated their responsibility to render spiritual care only, $57.14 \%$ rarely or never asses the patients spirituality background and $50 \%$ rarely or never gather information on the patient's spiritual background. It's been reported in literature that nurses who usually assess spiritual background of patients are more likely to practice and render high levels of spiritual care to their patients. ${ }^{15}$ The majority $(60 \%)$ also highlighted that they rarely or never pray or meditate with the patients. There sometimes exist a conflict between provision of care by the nurses and the need to respect the patient's privacy and freedom of religion and this can sometimes prevent nurses from engaging in spiritual activities that are deem to have a strong attachment effect such as praying or meditation. A study in South Africa also reported similar findings were nurses rarely participated in patients' spiritual rituals such as praying and meditation due to ethical constraints related to provision of spiritual care. $^{2}$ Whilst a majority of the participants $(53.33 \%)$ sometimes and often recommended meditation in this study, they rarely took part in the activity with the patient. Overall $50 \%$ of the participants indicated they refer patients to priests and other spiritual leaders for spiritual support. Even though, referring a patient is also part of providing spiritual care to patient, it can also regarded as transferring of duties by nurses. This was also a similarly reported among nurses in a previous study where $73 \%$ referred patients to the clergy or encourage them to pray on their own privately. Participants indicated that they rarely or never talk to patients about death or allowing them to talk about their death. They do not help patients reflect themselves on their beliefs of what happens after death. Death is a sensitive subject to discuss in the Africa culture and is usually avoided by those affected and caring for the patient. However, in a similar study nurses reported that they render nursing care to patients and relatives such as supporting them with end of life decisions, supporting them when feeling being lost and unbalanced, encouraging exploration of meaning of life, and providing space, time and privacy to talk ${ }^{3}$. The findings suggest that Participants are aware of spiritual care but are not largely in tune with rendering patient spiritual care. The findings of this study are in line with a similar study which reported that nurses' willingness to provide such care has rarely been reported. ${ }^{16}$

\section{Effectiveness of spiritual care given to patients by registered nurses}

Majority of the participants (62.5\%), indicated that prognosis was good for patients they offered spiritual care to. In addition, $6.25 \%$ indicated improving and $6.25 \%$ reported fairly. The findings showed that provision of spiritual care can have a positive impact to patients as it indicated a possible improvement in prognosis. Since the spirit is part of the person, attention to whole being, physical, mental and spiritual, can have a positive impact on patient disease outcomes and recovery.

\section{Conclusion}

All the participants participated in spiritual/religious activities often and majority of the participants belonged to the Christian faith. A majority of the registered nurses understood the concept of spiritual care and that the spirit is part of the body and without its consideration, provision of holistic care to patients is not possible. Registered nurses understand that it is their role to ensure that holistic care and spiritual care are rendered. However, majority of the participants rarely or never rendered spiritual care to patients. Only a few refereed patients to pastors and other religious leaders or recommend spiritual books and meditations. Spiritual care, where it was provided was reported to have a positive effect on the prognosis. Findings from this study indicated that spiritual care in an important part of holistic care however, there is need for guidelines in terms of provision of spiritual care by nurses.

\section{Acknowledgments}

None

\section{Conflict of interest}

The author declares that there is no conflict of interest.

\section{References}

1. Lundberg PC, Kerdonfag P. Spiritual care by Thai nurses in Intensive Care Units. J Clin Nurs. 2010;19(78):1121-1128.

2. Chandramohan S, Bhagwan R. (2015). Spirituality and spiritual care in in the context of nursing education in South Africa: original research. Curationis. 2015;38(1):1-15.

3. Nixon AV, Narayanasamy A, Vivian P. An investigation into the spiritual needs of neuro-oncology patients from a nurse perspective. BMC Nursing. $2013 ; 12: 2$.

4. O'Shea ER, Wallace M, Quinn Griffins M, et al. The effects of an educational session on paediatric nurses. J Pediatr Nurs. 2011:26(1):34 43.

5. Dunn L. Spirituality and nursing: Personal responsibility. Online Journal of Rural Nursing \& Health Care. 2008;8(1):3-4. 
6. Koenig HG. Research on religion, spirituality and mental health: A review. Canadian Journal of Psychiatry. 2009;54(5):283-291.

7. Monareng LV. An exploration of how spiritual nursing care is applied in clinical nursing practice. Health SA Gesondheid. 2013;18(1):1-8.

8. Collins P. Negotiating Selves: Reflections on "unstructured interviewing. 2006.

9. Burkhardt M, Nagai-Jacobson M. Spirituality and health. In: Dossey B, I Keegen, editors. Holistic nursing: A handbook for practice. Burlington, MA: Jones \& Bartlett; 2013.

10. Chiang YC, Lee HC, Chu TL, et al. The impact of nurses' spiritual health on their attitudes toward spiritual care, professional commitment, and caring. Nursing Outlook. 2016;64(3):215-224.

11. Beth D, Lee A, Jeremy FP, et al. Spiritual Care Practices of Advanced Practice Nurses: A Multinational Study. The Journal for Nurse Practitioners. 2016; 12(8):536-544.
12. Bailey ME, Moran S, Graham MM. Creating a spiritual tapestry of nurses' experiences of delivering spiritual care to patients in an Irish hospice. Int J Palliat Nurs. 2009;15(1):42-48.

13. Melhem GA, Zeilani RS, Zaqqout OA, et al. Nurses' Perceptions of Spirituality and Spiritual Care Giving: A Comparison Study Among All Health Care Sectors in Jordan. Indian J Palliat Care. 2016;22(1):42-49.

14. Li-Fen W, Hui-Chen C, Yu-Chen L. Nurse education and willingness to provide spiritual care. Nurse Education Today. 2016;38:36-41.

15. Gallison BS, Xu Y, Jurgens CY, et al. Acute care nurses' spiritual care practices. Journal of Holistic Nursing. 2013;31(2):95-103.

16. Wu LF, Tseng HC, Liao YC. Nurse education and willingness to provide spiritual care. Nurse Education Today. 2016;38:36-41. 